\title{
New Methods for MRI Denoising based on Sparseness and Self-Similarity
}

\author{
José V. Manjón ${ }^{a}$, Pierrick Coupéb ${ }^{b}$ Antonio Buades ${ }^{c, d}$, \\ D. Louis Collins ${ }^{b}$, Montserrat Robles ${ }^{a}$ \\ E-mail addresses:
}

jmanjon@fis.upv.es, pierrick.coupe@gmail.com, toni.buades@uib.es, louis.collins@mcgill.ca, mrobles@fis.upv.es

a Instituto de Aplicaciones de las Tecnologías de la Información y de las Comunicaciones Avanzadas (ITACA), Universidad Politécnica de Valencia, Camino de Vera s/n, 46022 Valencia, Spain

b McConnell Brain Imaging Centre, Montreal Neurological Institute, McGill University, Montreal, Canada

${ }^{c}$ Université Paris Descartes, 45 rue des Saints Pères, 75270 Paris Cedex 06, France

d Dpt Matemàtiques i Informàtica, Universitat Illes Balears, Ctra Valldemossa km 7.5, 07122 Palma de Mallorca, Spain.

* Corresponding author: José V. Manjón. Instituto de Aplicaciones de las Tecnologías de la Información y de las Comunicaciones Avanzadas (ITACA), Universidad Politécnica de Valencia, Camino de Vera s/n, 46022 Valencia, Spain

Tel.: (+34) 963877000 Ext. 75275, Fax: (+34) 963879009

E-mail: jmanjon@fis.upv.es (José V. Manjón) 


\section{Abstract}

This paper proposes two new methods for the three-dimensional denoising of magnetic resonance images that exploit the sparseness and self-similarity properties of the images. The proposed methods are based on a threedimensional moving-window discrete cosine transform hard thresholding and a three-dimensional rotationally invariant version of the well-known nonlocal means filter. The proposed approaches were compared with related state-ofthe-art methods and produced very competitive results. Both methods run in less than a minute, making them usable in most clinical and research settings.

Keywords: MRI, denoising, sparseness, nonlocal means

\section{Abbreviations:}

VBM: Voxel-based morphometry

PCA: Principal component analysis

DCT: Discrete cosine transform

NLM: Nonlocal means

ODCT3D: Oracle-based 3D discrete cosine transform filter

RI-NLM3D: Rotationally invariant nonlocal means filter

PRI-NLM3D: Prefiltered rotationally invariant nonlocal means filter

WSM: Wavelet sub-band mixing

RMSE: Root mean squared error

SSIM: Structural similarity index 


\section{Introduction}

The denoising of magnetic resonance (MR) images is an important open problem that has been discussed frequently in the recent literature because of its relevance to many clinical and research uses. Denoising is used as a preprocessing step in many image processing and analysis tasks such as registration or segmentation to reduce the random noise arising from the acquisition process.

One approach that has been applied extensively in MRI preprocessing is the Gaussian filter (Ashburner and Friston, 2000). This method, although capable of reducing some image noise (especially in homogeneous areas), also removes high-frequency signal components, thereby blurring edges in the images. Therefore, this filter has been commonly used for regularization purposes, such as in voxel-based morphometry (VBM) (Ashburner and Friston, 2000), to reduce anatomical inconsistencies.

A large number of edge-preserving methods have been proposed to overcome the above-mentioned blurring effects. For example, anisotropic diffusion filters (Gerig et al., 1992) are able to remove noise using gradient information while respecting important image structures. Recently, Krissian and Aja-Fernandez (2009) proposed a new anisotropic diffusion filter based on a linear minimum mean squared error estimation and partial difference equations for Rician noise removal that has achieved state-of-the-art results.

Wavelet-based filters have also been applied successfully to the denoising of MR images (Pizurica et al., 2003). Such filters are rooted in the processing of images in a transformed domain. Other transforms that have been applied to denoise images include principal component analysis (PCA) (Muresan and Parks, 2003) and the discrete cosine transform (DCT) (Yaroslavsky et al., 2000). Many transform domain filters derive from variations of the transformthreshold-inverse transform principle. 
In accordance with this principle, local transform approaches (i.e., sliding window with or without overlapping) have obtained very good results in recent years (Guleryuz, 2003; Guleryuz, 2007; Yaroslavsky et al., 2000). In Guleryuz's (2007) method for Gaussian noise reduction, the image noise is removed using overcomplete linear transforms and thresholding. In practice, Guleryuz applied a classical sliding-window DCT thresholding as in Yaroslavsky et al. (2000), but overlapping estimations were adaptively combined to reduce the Gibbs effects.

Other recently proposed approaches use learned image patch dictionaries (Aharon et al., 2006; Elad and Aharon, 2006; Mairal et al., 2008) instead of DCT bases to perform the denoising. All these approaches stem from the fact that an image can be represented as the linear combination of a set of image bases with very few non-null coefficients. This property, known as sparseness, is the core of the JPEG and JPEG2000 compression standards.

Finally, the nonlocal means (NLM) filter, a new method introduced by Buades et al. (2005), has emerged as a very simple and effective way to reduce noise while minimally affecting the original structures of the image. This method is based on the natural redundancy of patterns within the images. Recently improved, the NLM filter has been applied to the denoising of MR images and demonstrated better results than those of previous related methods (Coupé et al., 2008a, 2008b; Manjón et al., 2010; Wiest-Daesllé et al., 2008).

In this paper, we present two new approaches to the three-dimensional (3D) denoising of $M R$ images. The first is an extension of the original method proposed by Guleryuz (2007). Based on local 3D DCT hard thresholding, our proposed method has been adapted to deal with Rician noise (the typical type of noise present in magnitude MR images) using a pseudo-oracle principle. The second proposed method is a new rotationally invariant 3D version of the Rician-adapted NLM filter that uses a prefiltered image obtained by DCT denoising. 


\section{Methods}

Typically, the observed noisy MR image $y$ is considered the linear combination of a noise-free image $x$ and a white noise realization $n$ from the measurement process:

$$
y=x+n
$$

Therefore, the goal of any denoising algorithm is to find a good estimate $\hat{x}$, given $y$. This section describes two recent methods for image denoising based on efficient concepts of how to solve this problem:

- Denoising using sparseness: The first method under study is the local DCT denoising method proposed by Guleryuz (2007) that uses the sparseness of the image (i.e., the ability of the image to be represented by a small number of base functions, e.g., DCT bases).

- Denoising using self-similarity: The second method under investigation is the well-known NLM filter proposed by Buades et al. (2005) that uses the pattern redundancy present in the image. After a brief presentation of the original NLM filter, a new rotationally invariant version of this filter is proposed.

In order to take advantage of both properties, sparseness and self-similarity, a new efficient collaborative method is proposed. The proposed technique is based on a rotationally invariant version of the NLM filter and uses an image prefiltered with the extended DCT-based method to compute patch similarities.

Finally, the adaptation of the proposed methods to allow them to handle Rician noise (i.e., the type of noise present in magnitude MR images) is described. 


\subsection{Denoising using sparseness}

\subsubsection{Guleryuz's method (DCT3D)}

In Guleryuz's (2007) method, the estimate of the noise-free image $\hat{x}$ is obtained using a hard thresholding method. An overcomplete set of 3D block DCTs $(4 \times 4 \times 4$ block size) is used (Guleryuz used $8 \times 8$ blocks in his $2 D$ method, which yields the same number of coefficients). The local denoised estimate at block $j$, $\hat{x}_{j}$, is obtained by applying a hard thresholding rule:

$$
c_{j}=\mathrm{H}\left(y_{j}\right) \quad \hat{c}_{j}=T\left(c_{j}, \tau\right) \quad \hat{x}_{j}=H^{-1}\left(\hat{c}_{j}\right)
$$

where $\mathrm{H}$ is a 3D DCT, $c_{j}$ are the transform coefficients of the $j$ block, and $T$ is the hard thresholding operator with threshold $\tau$. Finally, all local estimates $\hat{x}_{j}(i)$ are combined from all overlapping $j$ blocks at position $i$ using the following weighted average rule:

$$
\hat{x}(i)=\frac{\sum_{j=1}^{V} \theta_{j} \hat{x}_{j}(i)}{\sum_{j=1}^{V} \theta_{j}} \quad \theta_{j}=\frac{1}{1+\left\|\hat{c}_{j}\right\|_{0}}
$$

where $V$ is the number of overlapping blocks contributing to $\hat{x}_{(i)}$ and $\theta_{j}$ is the weight of each block $j$, which is proportional to the inverse of the $\hat{c}_{j} L O$ norm (number of nonzero coefficients of block $j$ after the thresholding operation). This approach gives more weight to estimations with more null values after thresholding. Thus, homogeneous blocks tend to be favored in comparison with blocks containing edges and thus suffering from Gibbs effects. The only parameter in this method is the threshold value $\tau$. 


\subsubsection{Oracle-based filters}

Oracle-based filters assume that, if the null coefficients of the original noise-free image are known, they can be applied to improve the denoising. In fact, the thresholding method that minimizes the mean squared error with the original image is given by:

$$
p_{j}=H\left(x_{j}\right) \quad \hat{c}_{j}(i)=\left\{\begin{array}{l}
0 \quad \text { if }\left(\left|p_{j}(i)\right|<\sigma\right) \\
c_{j}(i) \text { otherwise }
\end{array}\right.
$$

which compares the magnitude of the noise-free DCT coefficients with the standard deviation of the noise $\sigma$ (see Mallat (1999) for more details).

Unfortunately, in practice, no noise-free image $x$ is available; hence, this approach is unfeasible.

\subsubsection{Proposed Oracle-based DCT filter (ODCT3D)}

If we relax the Oracle condition, we can use a prefiltered image $\hat{x}^{\text {pre }}$ with, for example, the described DCT3D method as an approximation of the noise-free image. With this approach, the $c_{j}$ block coefficients of the noisy image $y$ can be better thresholded (applying the threshold operation described in Equation 4) using the corresponding block coefficients $p_{j}$ of the prefiltered image. The final image reconstruction is performed using the same approach described above. We will refer to this method as Oracle DCT3D (ODCT3D). As we will show later, this modification not only allows better results to be obtained in terms of quantitative measures, but also reduces the remaining Gibbs artifacts of the original DCT3D method.

The only parameter of this method is the threshold $\tau$ from the prefiltering step. In all of our experiments, we used a $\tau$ value of $2.7 \sigma$ (where $\sigma$ is the standard deviation of the noise), which is common in DCT and wavelet thresholding methods (Mallat, 1999). 


\subsection{Denoising using self-similarity}

\subsubsection{The original nonlocal means denoising filter}

For its use of the self-similarity properties of an image, the NLM filter has recently become known as a simple but effective way of removing noise from an image while minimally affecting its original structure.

Originally proposed by Buades et al. (2005), the NLM filter takes advantage of the high level of pattern redundancy in an image, achieving high-quality image denoising by averaging similar realizations of the noisy signals. Basically, this filter reduces the noise in an image by averaging voxels that originally had the same intensity in the noise-free image. To this end, Buades et al. (2005) suggested that voxels with similar neighborhoods (small 3D patches in our volumetric case) tend to have similar original values, yielding the following filter expression:

$$
\hat{x}(i)=\frac{\sum_{j \in \Omega} w(i, j) y(i)}{\sum_{j \in \Omega} w(i, j)} \quad w(i, j)=e^{-\frac{\left\|N_{i}-N_{j}\right\|_{2}^{2}}{h^{2}}}
$$

where $\Omega$ represents the search volume, the weights $w(i, j)$ represent the similarity between any two 3D patches $N_{i}$ and $N_{j}$ centered around pixels $i$ and $j$, and $h^{2}$ is a parameter controlling the strength of the filter.

So defined, this filter is able to identify $N_{j}$ patches as similar to a given patch $N_{i}$, such that they share the same structure and orientation. This means that the weights defined in Equation 5 are not rotationally invariant. Therefore, similar patches with similar structure but different orientations to the reference patch will have a small influence in the average. 


\subsubsection{Rotationally invariant nonlocal means filter (RI-NLM3D)}

To avoid this problem, the orientation of each patch can be estimated and corrected before computing the $\mathrm{L} 2$ norm (Kleinschmidt et al., 2008; Zimmer et al., 2008). However, this solution is very time consuming, as it increases the already high computational burden of the NLM method, and the reorientation operation introduces interpolation artifacts and correlates the noise, making the denoising process more difficult.

Instead of correcting the orientation of the patches, a rotationally invariant measure describing the patch information can be used (Lou et al., 2008; Zexuan et al., 2009), for instance, rotationally invariant image descriptors such as $\mathrm{Hu}$ or Zernike moments (Hu, 1962; Teague, 1980). However, such descriptors are sensitive to noise, and their ability to represent patch information decreases rapidly as noise power increases.

Therefore, we present a simple but effective similarity measure that is rotationally invariant and based on voxel intensity and the corresponding local patch mean (using a $3 \times 3 \times 3$ voxel Gaussian kernel):

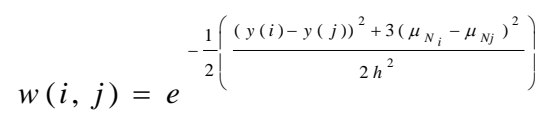

where $\mu_{N i}$ and $\mu_{N i}$ are the mean values of patches $N_{i}$ and $N_{j}$ around voxels $i$ and $j$, and $h$ is related to the standard deviation of the noise. Note that the distance between the patch means is multiplied by three to account for this distance being approximately three times smaller than the average voxel distance because of the application of Gaussian smoothing. Our experiments showed that giving this distance three times the weight produces the best results.

Because it uses the value of the central voxel of the patch, this similarity measure is point specific rather than region specific, which allows a better description of the characteristics of the voxel. On the other hand, using the mean value of the region around the voxel would make the similarity measure 
more robust against the noise while minimizing the aggregation artifacts that could produce cartoon-like images.

In analyzing Equation 6, it becomes clear that this measure exhibits the same noise sensitivity as Hu or Zernike moments because it uses the intensity of the voxel of the similarity measure, which is not a robust descriptor of the real intensity of the voxel (the main benefit of this approach is its low computational complexity). For this reason, applying this filter to medium and high noise levels produces poor results. However, we will show that, under certain circumstances, this method can obtain very good denoising results when combined with a prefiltering step. We will refer to this method as the RI-NLM3D.

\subsection{A new collaborative method based on sparseness and self-similarity}

We propose to combine the two methods described above to obtain better results than could be obtained by either method separately. Specifically, we propose an Oracle-based RI-NLM3D method where the similarities between voxels and patches are computed from the already denoised image using the ODCT3D method and then applied to denoise the original noisy image:

$$
\hat{x}(i)=\frac{\sum_{j \in \Omega} \beta(i, j) y(i)}{\sum_{j \in \Omega} \beta(i, j)} \quad \beta(i, j)=e^{-\frac{1}{2}\left(\frac{\left(\hat{x}^{o}(i)-\hat{x}^{o}(j)\right)^{2}+3\left(\mu_{N_{i}}^{o}-\mu_{N j}^{o}\right)^{2}}{2 h^{2}}\right)}
$$

where the new weight $\beta$ is computed using the ODCT3D denoised image $x^{\circ}$ and applied to denoise the noisy image $y$.

Kervrann et al. (2007) proposed a similar plug-in estimator for 2D NLM filtering. In their approach, they first denoised the image with a Bayesian NLM filter. The similarity between denoised patches was then computed and finally applied to noisy voxels. However, this method has the disadvantage of being computationally intensive and only based on pattern redundancy without taking into consideration the sparseness properties of the image. 
By contrast, the proposed approach presents an interesting advantage in that the RI-NLM3D method only works well for low noise levels, a condition that is met after the ODCT3D method has been applied. Figure 1 shows a scheme of the proposed method.

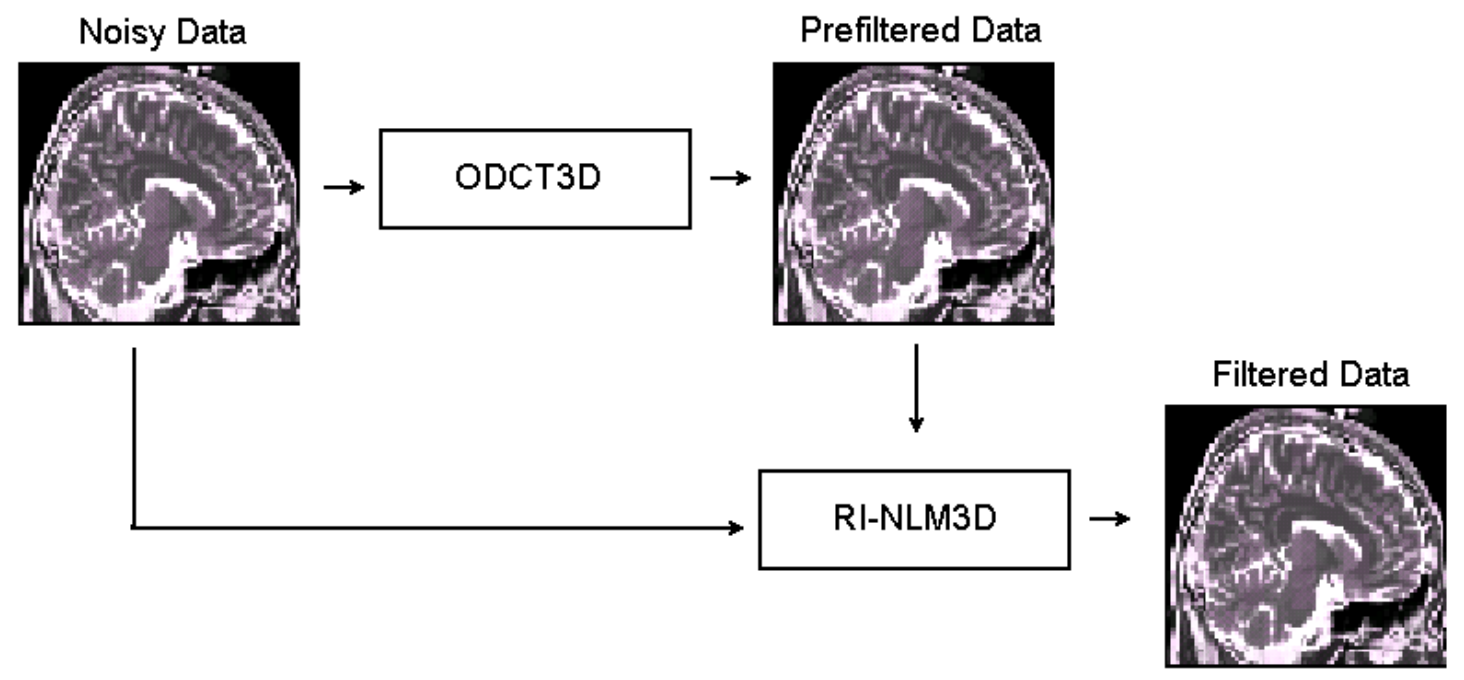

Fig. 1. Scheme of the proposed method.

Finally, a preselection strategy was also applied to avoid useless computations (Coupé et al., 2008a; Kervrann et al., 2007). We used a statistically driven rule based on the distance between the patch means of the prefiltered volume:

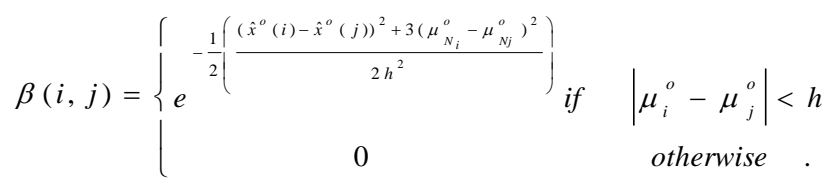

In this way, patches with an intensity difference greater than $h$ do not contribute to the denoising process. We will refer to this proposed method as the prefiltered rotationally invariant NLM3D (PRI-NLM3D).

Because the proposed similarity measure is applied to the already denoised image using the ODCT3D method, the $h$ parameter controlling the strength of the filter was set to $0.4 \sigma$ instead of $\sigma$ (this value was found experimentally to be the best option through an exhaustive search of many image types and noise conditions). As in Coupé et al. (2008a), the radius of the search volume $\Omega$ was 
set to 5 voxels, which resulted in a $3 D$ search volume of $11 \times 11 \times 11$ voxels. Experimentally, this volume was shown to be a good compromise between computational complexity and quality of the results. Larger search volumes improved the results only marginally, but increased the computational burden significantly.

\subsection{Adaptation to Rician noise}

Noise in magnitude MR images follows a Rician distribution (Nowak, 1999). Consequently, the weighted average produced by applying the PRI-NLM3D method will be biased because of the asymmetry of the Rician distribution.

To avoid such bias, we adopted a method of bias correction used in early MRI denoising (Manjón et al., 2008; Wiest-Daesslé et al., 2008). The unbiased PRINLM3D estimator is defined as follows:

$$
\hat{x}(i)=\sqrt{\left.\max \left\{\mid \frac{\sum_{j \in \Omega_{i}} \beta(i, j) y(i)^{2}}{\sum_{j \in \Omega_{i}} \beta(i, j)}\right)-2 \sigma^{2}, 0\right)}
$$

We also adapted the ODCT3D method to deal with Rician noise. In this case, because of the effect of DCT thresholding, the bias in the squared domain is not constant, but dependent on intensity. It can be estimated theoretically and inverted in the original domain using the properties of the first moment of a Rician distribution, as expressed in Equation 10:

$$
\begin{gathered}
\hat{\mathrm{x}}_{j}(\mathrm{i})=\frac{\pi}{2} \times \exp \left(-\frac{\phi_{j}(i)^{2}}{2}\right)\left(\left(1+\frac{\phi_{j}(i)^{2}}{2}\right) I_{0}\left(\frac{\phi_{j}(i)^{2}}{4}\right)+\left(\frac{\phi_{j}(i)^{2}}{2}\right) I_{1}\left(\frac{\phi_{j}(i)^{2}}{4}\right)\right)^{2} \\
\text { with } \phi_{j}(i)=\frac{x_{j}(i)}{\sigma}
\end{gathered}
$$

where $\hat{x}_{j}(i)$ and $x_{j}(i)$ are the biased and unbiased estimates due to the Rician bias at position $i$ from block $j, I_{0}$ and $I_{1}$ are the modified Bessel functions of order 
zero and one, respectively, and $\sigma$ is the standard deviation of the noise. Then, the final unbiased estimate is obtained by mapping the biased estimates directly onto the unbiased ones by using a precomputed look-up table. Finally, the different estimations from the different blocks are averaged to provide the final unbiased estimate in a manner similar to Equation 3:

$$
\hat{x}(i)=\sqrt{\frac{\sum_{j=1}^{V} \theta_{j} \eta\left(\hat{x}_{j}(i)\right)}{\sum_{j=1}^{V} \theta_{j}}}
$$

where operator $\eta$ is a mapping function that provides the unbiased estimate for a given biased estimate by using the precomputed equivalences from Equation 10.

\section{Experiments and results}

To evaluate the described methods, we used the well-known BrainWeb 3D MRI phantoms (Collins et al., 1998; Kwan et al., 1999), T1-weighted (T1w), PDw, and T2w volumes of $181 \times 217 \times 181$ voxels (voxel resolution $=1 \mathrm{~mm}^{3}$ ), which were corrupted with different levels of Rician noise (1\% to $15 \%$ of maximum intensity). Rician noise was generated by adding Gaussian noise to real and imaginary parts and then computing the magnitude image.

Two quality measures were used to evaluate the results. The first was the root mean squared error (RMSE) metric, which is a distance measure commonly used in image processing. The second was the structural similarity index (SSIM) (Wang et al., 2004), which is a measure more consistent with the human visual system:

$$
\operatorname{SSIM}(x, y)=\frac{\left(2 \mu_{x} \mu_{y}\right)\left(2 \sigma_{x y}+c_{2}\right)}{\left(\mu_{x}^{2}+\mu_{y}^{2}+c_{1}\right)\left(\sigma_{x}^{2}+\sigma_{y}^{2}+c_{2}\right)},
$$

where $\mu_{x}$ and $\mu_{y}$ are the mean value of images $x$ and $y, \sigma_{x}$ and $\sigma_{y}$ are the standard deviation of images $x$ and $y, \sigma_{x y}$ is the covariance of $x$ and $y$, 
$c_{1}=\left(k_{1} L\right)^{2}$, and $c_{2}=\left(k_{2} L\right)^{2}$ (where $L$ is the dynamic range, $k_{1}=0.01$ and $k_{2}=0.03$ ). As suggested by Wang et al. (2004), the SSIM was locally estimated using a Gaussian kernel of $3 \times 3 \times 3$ voxels. Finally, the mean value of all the local estimations was used as a quality metric. For the sake of clarity, both measures were estimated only in the region of interest (head tissues) obtained by removing the background (i.e., the label 0 of the discrete model in BrainWeb).

\subsubsection{DCT3D vs. ODCT3D}

To evaluate the proposed ODCT3D method, we compared it with the 3D version of Gurelyuz's (2007) method (DCT3D) using the T1w BrainWeb phantom. Figure 2 shows the RMSE and SSIM measures for different levels of Rician noise and indicates that the proposed method outperforms the DCT3D method at medium and high noise levels. Furthermore, as can be seen in Fig. 3, the proposed ODCT3D method reduces the Gibbs effects that remain after the DCT3D method is applied.
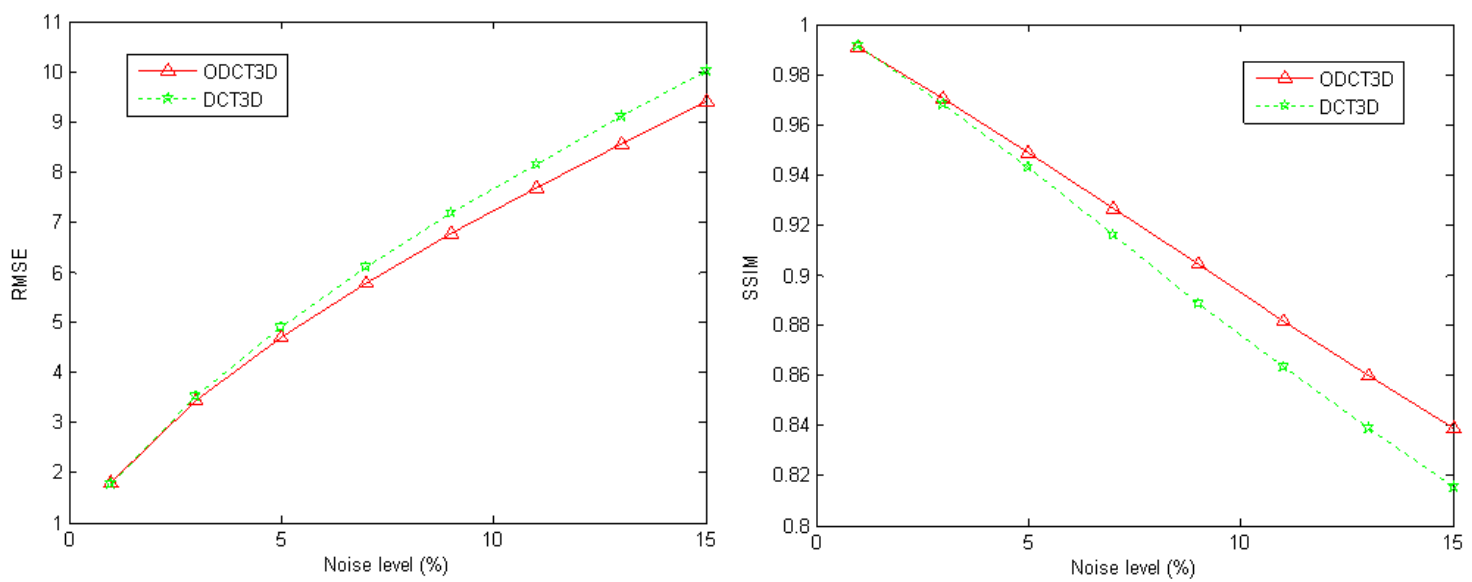

Fig. 2. Left: Root mean squared error (RMSE) of the ODCT3D and DCT3D methods for different noise levels. Right: Structural similarity index (SSIM) of the ODCT3D and DCT3D methods for different noise levels. 

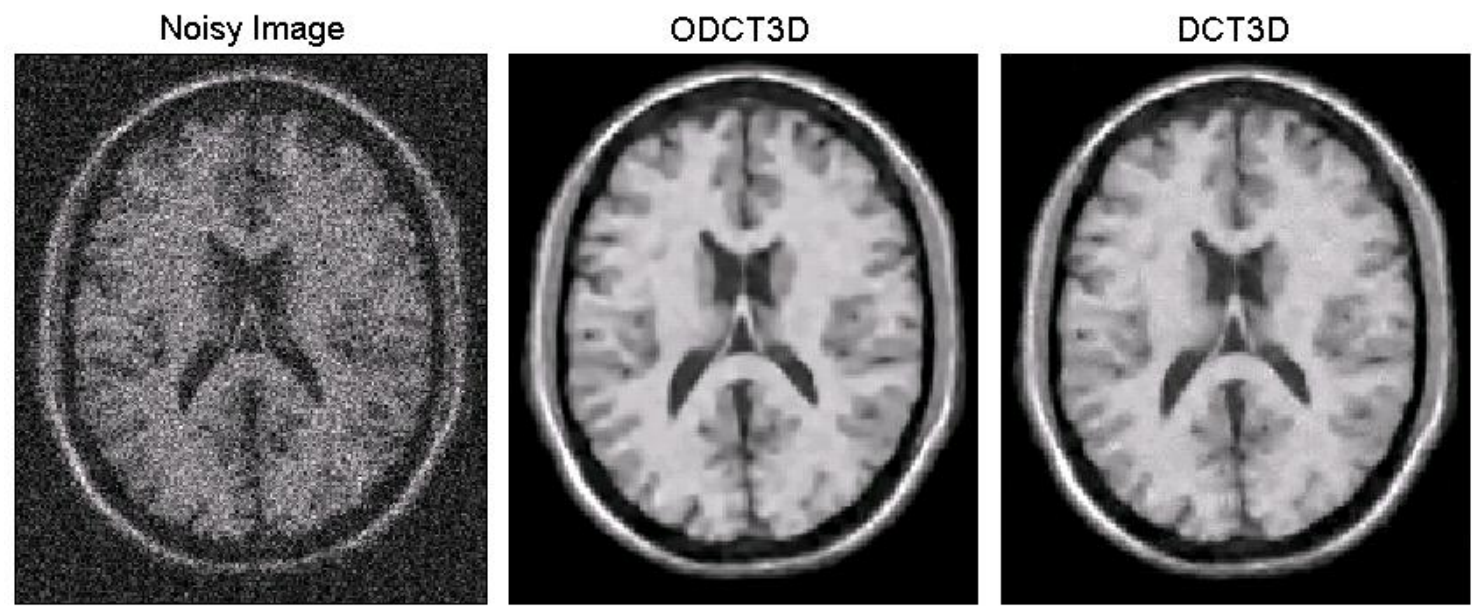

Fig. 3. Example result of the filtering process (axial slice) using the ODCT3D and DCT3D methods for a Rician noise level of $15 \%$. The DCT3D filtered image shows some Gibbs effects, mainly observable in homogeneous areas, while the image produced with the proposed ODCT3D method no longer shows such artifacts.

\subsection{Comparison of methods}

We compared our proposed Rician-adapted versions of the ODCT3D and PRINLM3D methods with some recently proposed related methods used for MRI denoising of Rician distributed noise: the optimized blockwise nonlocal means filter (Coupé et al., 2008a), the wavelet sub-band coefficient mixing method (WSM) (Coupé et al., 2008b), and the ORNRAD filter (Krissian and AjaFernandez, 2009).

Figure 4 shows a comparison of the RMSE and SSIM measures obtained using the methods under investigation with different image types and noise levels. Figures 5, 6, and 7 are provided for a visual comparison of the results. All the methods were run with the optimal parameters suggested by the authors (see Table 1). With the exception of the ORNRAD filter, all the methods were implemented using multithreaded C MEX files in MATLAB 2009a (The MathWorks, Inc.) in Windows XP 64-bit Edition (Pentium quad-core $2.4 \mathrm{GHz}$ with $8 \mathrm{~GB}$ of RAM). The ORNRAD filter was run (using AMILab 2.0.4 on an Intel Xeon X5650 with 12 cores and 12 GB of RAM running Linux Fedora 13) by its authors, who supplied results for the same datasets. 
To facilitate the reproducibility of the presented experiments, the Matlab code for our the experiments will be available on our webpage: http://personales.upv.es/jmanjon/denoising/prinlm.html.

Table 1. Method parameters ( $v$ is the radius of the search volume, $f$ is the radius of the 3D patches, $h$ is the strength of the filter, and $\sigma$ is the standard deviation of the noise).

\begin{tabular}{cc}
\hline Method & Parameters \\
\hline Blockwise NLM & $v=5, \mathrm{f}=1, \mathrm{~h}=\sigma$ \\
WSM & $\mathrm{v}=3, \mathrm{f}_{1}=1, \mathrm{f}_{2}=2, \mathrm{~h}=\sigma$ \\
ODCT3D & $\tau=2.7 \sigma$ \\
PRI-NLM3D & $\mathrm{v}=5, \mathrm{~h}=0.4 \sigma$ \\
ORNRAD & $\mathrm{v}=1, \mathrm{~T}=2, \mathrm{dt}=1 / 6, \sigma_{1}=0.7, \sigma_{2}=1$ \\
\hline
\end{tabular}



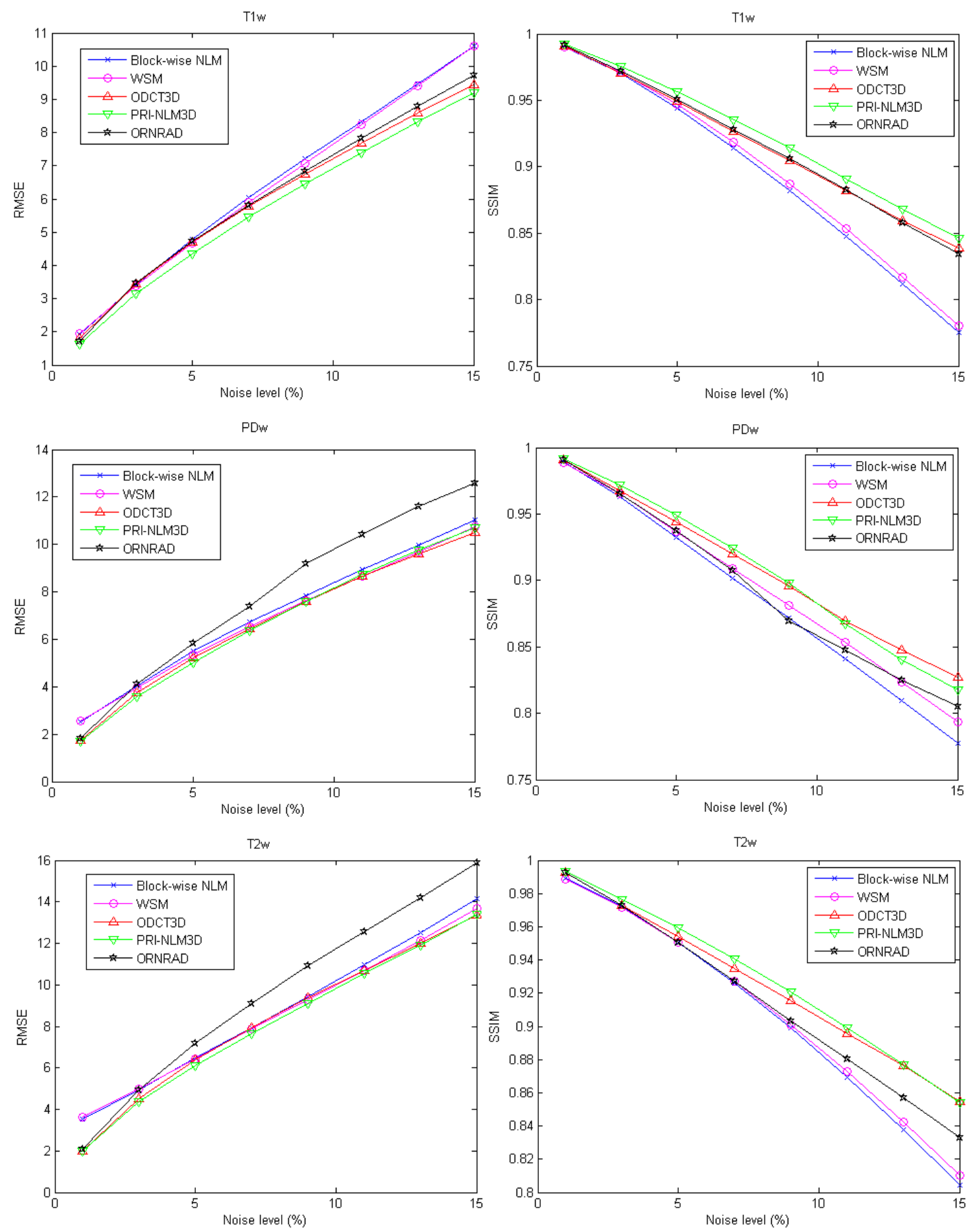

Fig. 4. Left: RMSE of the compared methods for different image types and noise levels. Right: SSIM of the compared methods for different image types and noise levels. 
As can be seen in Fig. 4, the proposed ODCT3D and PRI-NLM3D methods showed improvements over the other filters under consideration in most of the cases. The PRI-NLM3D method was the best method in almost all cases. Only the ODCT3D method surpassed the PRI-NLM3D method at high noise levels on the PDw data, probably because of the low contrast of the images. The ORNRAD method performed very well on the T1w images, producing similar results to those of the proposed ODCT3D method, although its performance decreased noticeably on the PDw and T2w images in terms of the RMSE.

Furthermore, as can be observed in Figs. 5, 6, and 7, the PRI-NLM3D produced visually more pleasant results than the other methods, showing fewer oscillations in homogeneous areas and better defined edges.

In terms of filtering time, the ORNRAD method was the slowest, taking $12 \mathrm{~min}$ on average (note that this was using a single-threaded implementation, whereas the other methods were running on four cores), followed by the WSM method at $110 \mathrm{~s}$, the blockwise NLM3D at $81 \mathrm{~s}$, the PRI-NLM3D method at $60 \mathrm{~s}$, and finally the ODCT3D method at only $10 \mathrm{~s}$. 


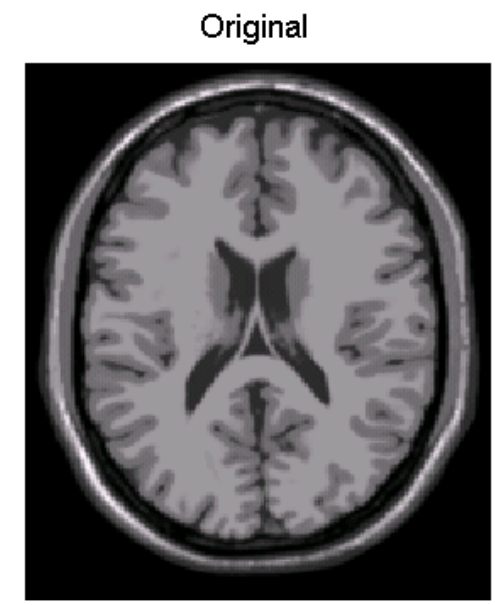

ORNRAD

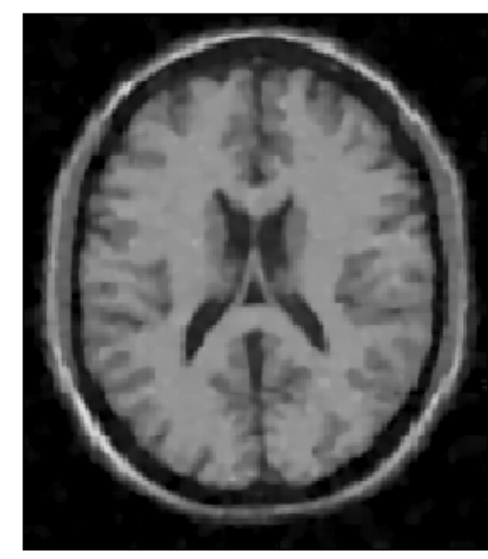

ORNRAD Residuals

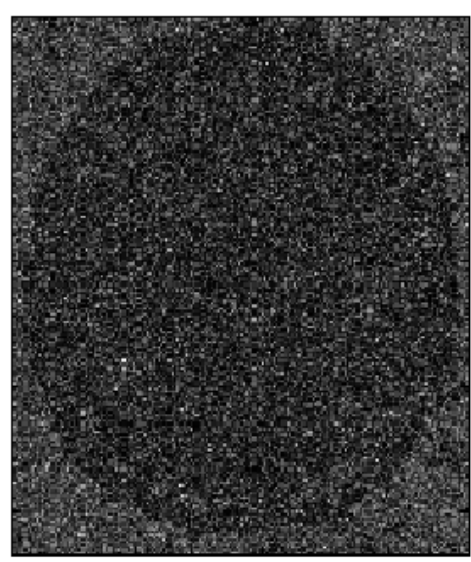

Noisy Image

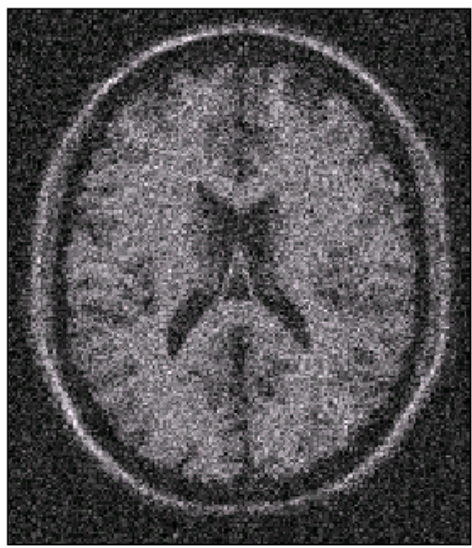

ODCT3D

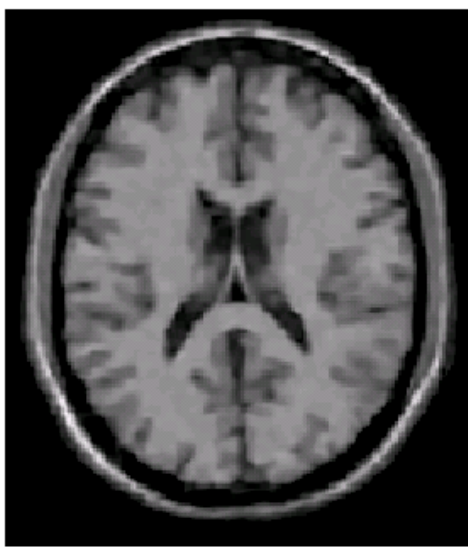

ODCT3D Residuals

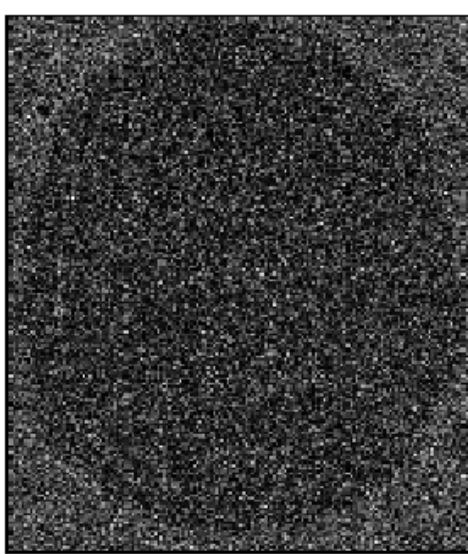

WSM

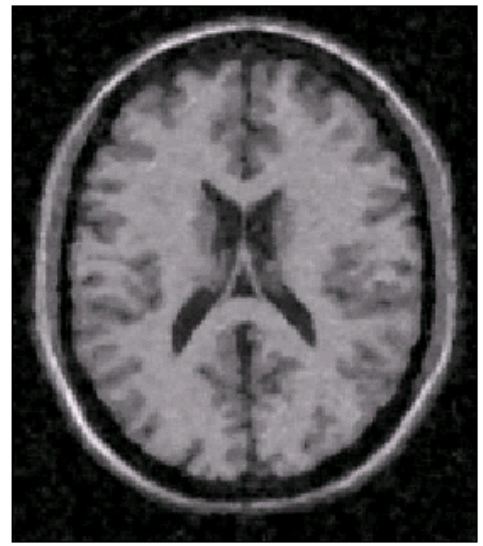

PRI-NLM3D

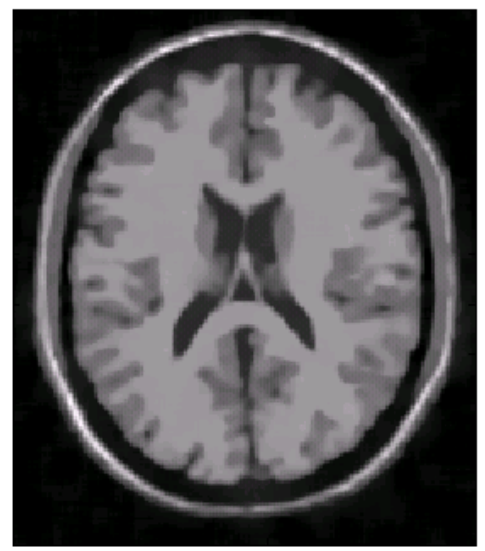

PRI-NLM3D Residuals

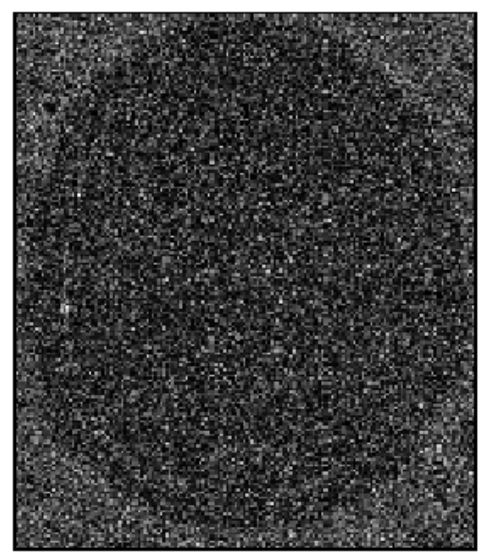

Fig. 5. Example filtering results for an axial slice of the T1w BrainWeb phantom (Rician noise level of $15 \%$ ). The third row shows the absolute value of the image residuals for the different methods. 


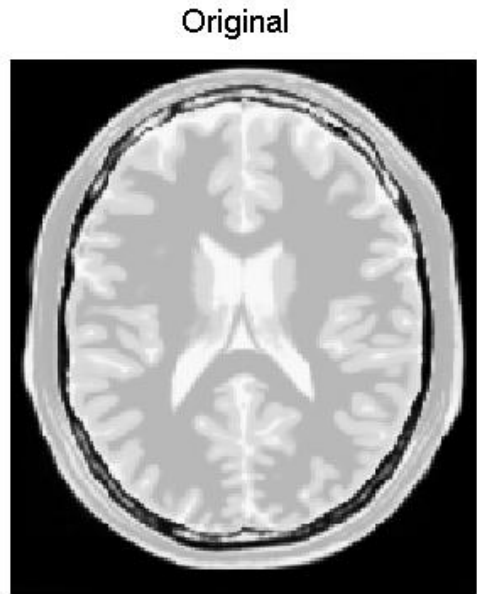

ORNRAD

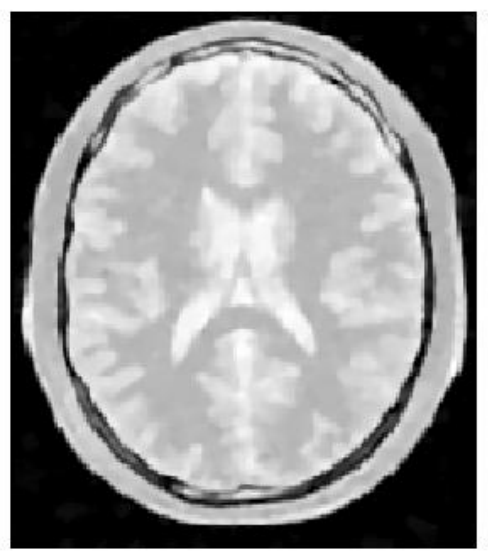

ORNRAD Residuals

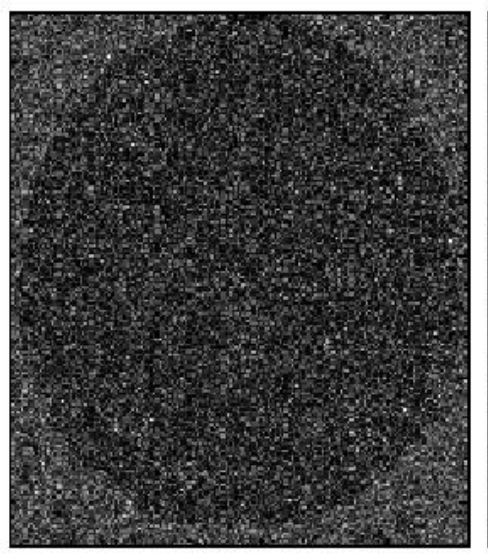

Noisy Image

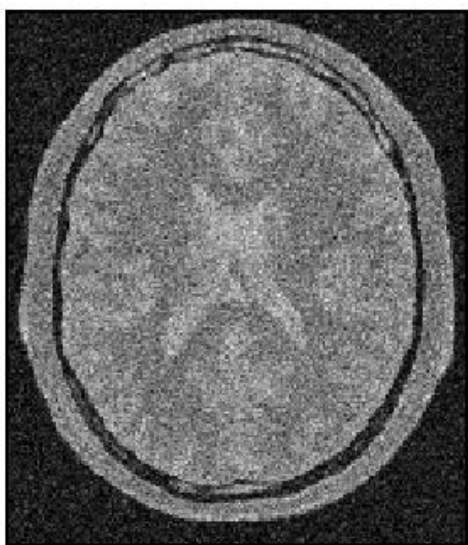

ODCT3D

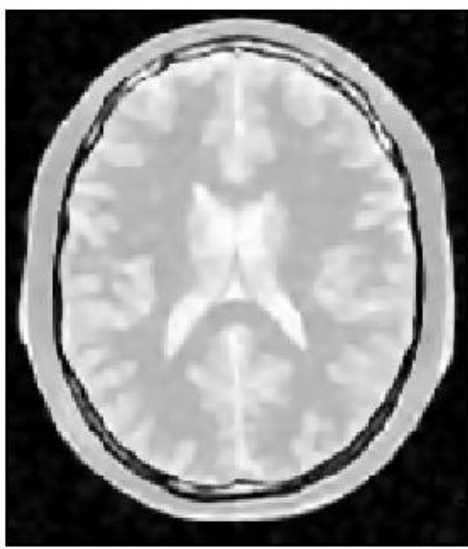

ODCT3D Residuals

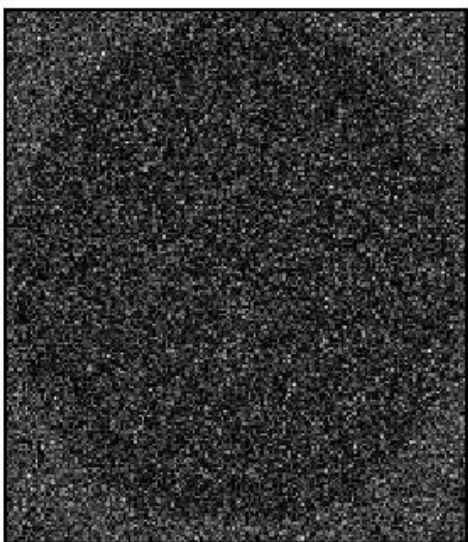

WSM

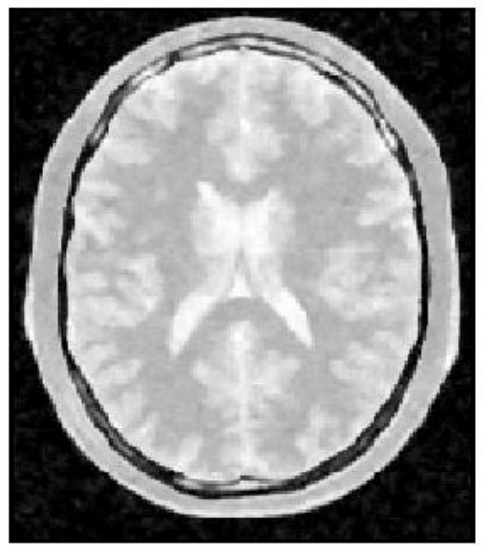

PRI-NLM3D

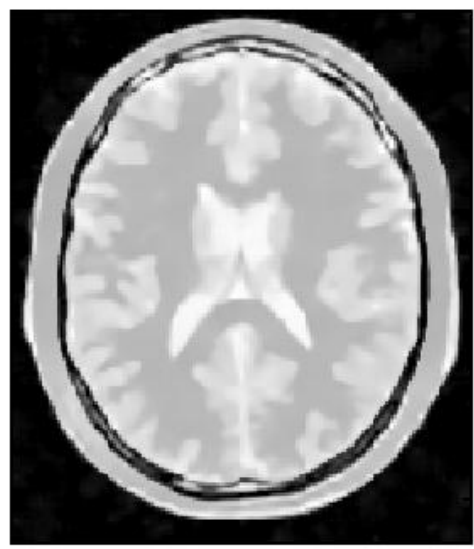

PRI-NLM3D Residuals

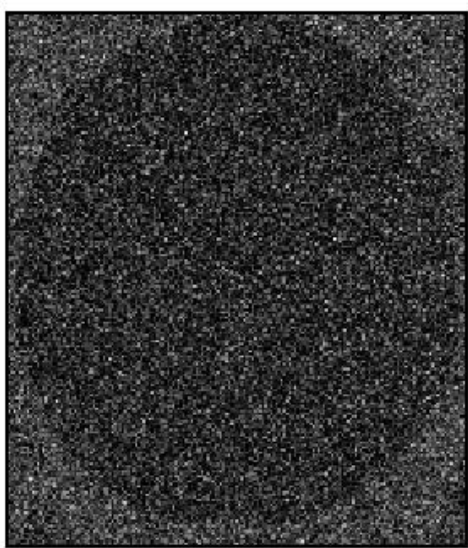

Fig. 6. Example filtering results for an axial slice of the PDw BrainWeb phantom (Rician noise level of $15 \%$ ). The third row shows the absolute value of the image residuals for the different methods. 


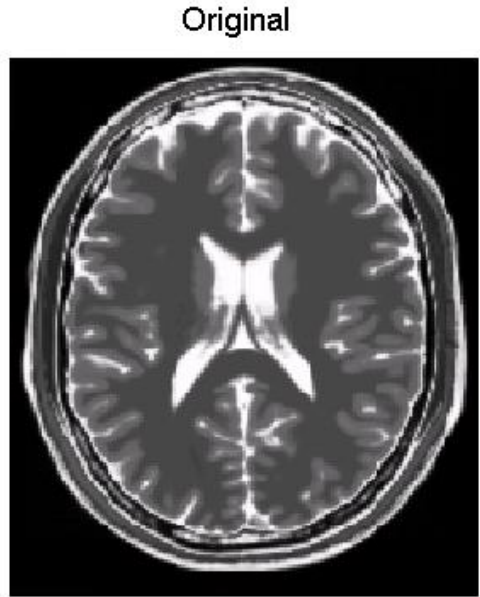

ORNRAD

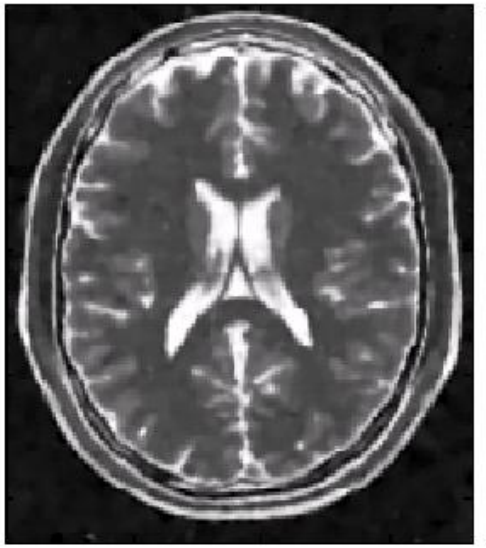

ORNRAD Residuals

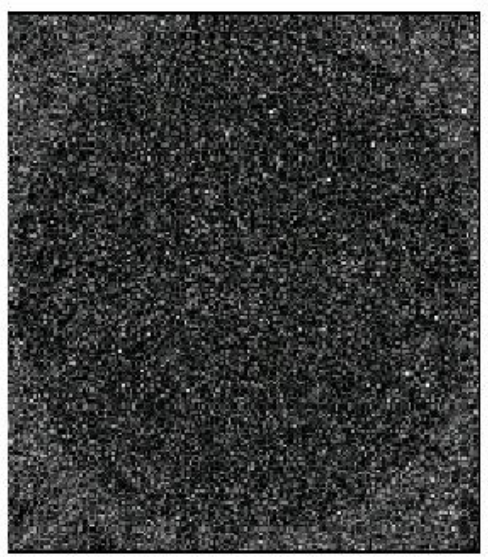

Noisy Image

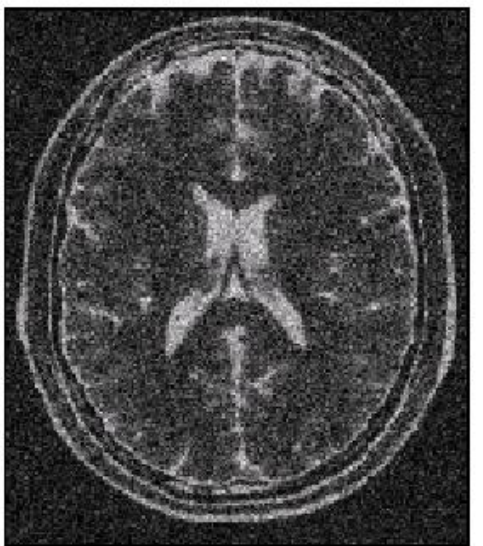

ODCT3D

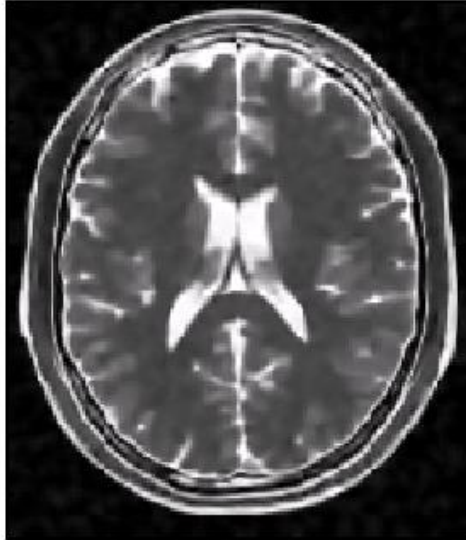

ODCT3D Residuals

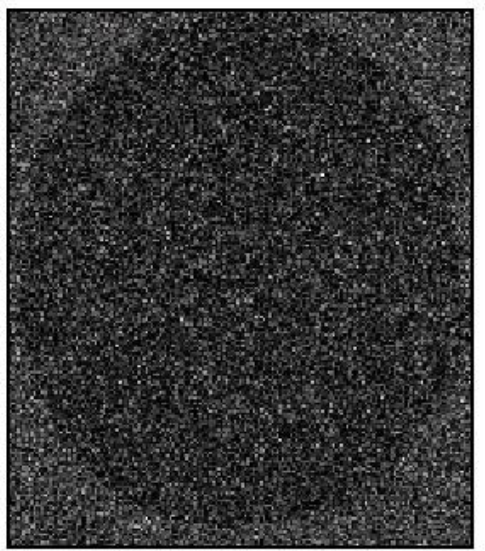

WSM

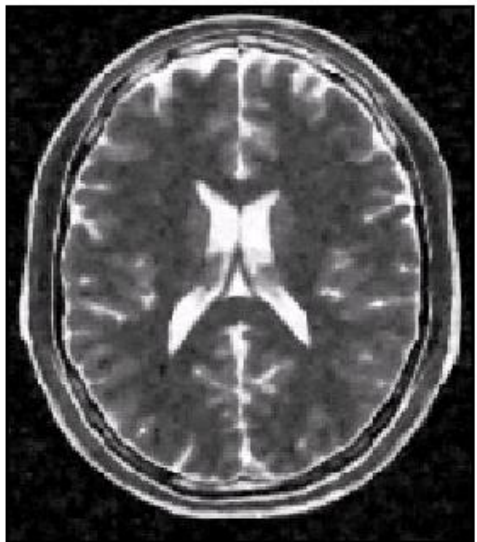

PRI-NLM3D

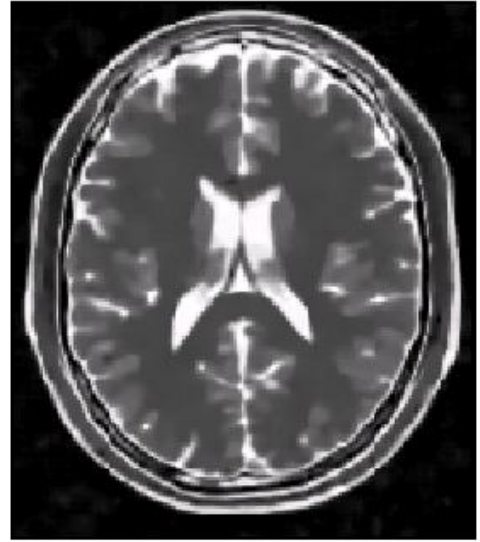

PRI-NLM3D Residuals

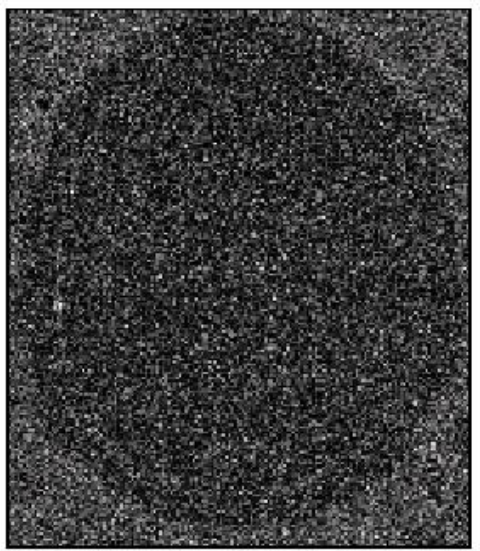

Fig. 7. Example filtering results for an axial slice of the T2w BrainWeb phantom (Rician noise level of $15 \%$ ). The third row shows the absolute value of the image residuals for the different methods. 


\subsection{Evaluation on real clinical data}

To evaluate the consistency of the PRI-NLM3D method on clinical data, two datasets were used. The first was an MP-RAGE T1w volumetric sequence acquired on a Siemens 1.5T Vision scanner (Erlangen, Germany) [TR $=9.7 \mathrm{~ms}$,

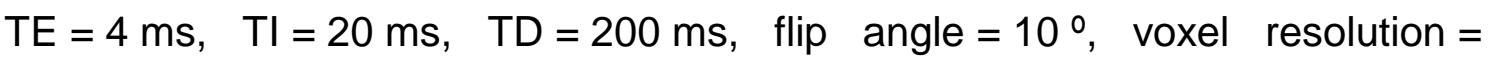
$1 \times 1 \times 1.25 \mathrm{~mm}^{3}, 256 \times 256 \times 128$ voxels]. The Rician noise level (used as the filtering parameter) was estimated to be around $2 \%$ of the maximum intensity using the object-based method proposed by Coupé et al. (2010). The filtering results for this dataset are shown in Fig. 8. The PRI-NLM3D method removed the noise successfully, showing no significant anatomical information on the image residuals. The processing time for this dataset was $42 \mathrm{~s}$.

The second dataset was an MP-RAGE T1w volumetric sequence acquired on a Siemens Trio 3T scanner (Erlangen, Germany) $[\mathrm{TR}=2300 \mathrm{~ms}$, TE $=2.9 \mathrm{~ms}$,

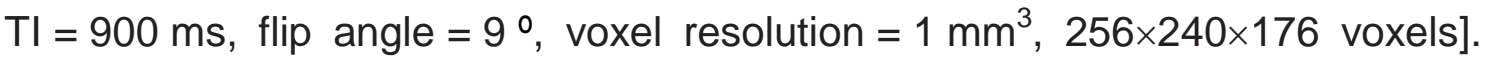
The Rician noise level was estimated to be around $1 \%$ of maximum intensity. Figure 9 allows for a visual comparison of the results produced using the ODCT3D, ORNRAD, and PRI-NLM3D methods. The PRI-NLM3D method removed the noise successfully while preserving fine details of the images, whereas the ODCT3D and especially the ORNRAD method slightly oversmoothed some details. The processing time for this dataset was $9 \mathrm{~s}$ with the ODCT3D method, $52 \mathrm{~s}$ with the PRI-NLM3D method, and $13 \mathrm{~min}$ with the ORNRAD filter. 


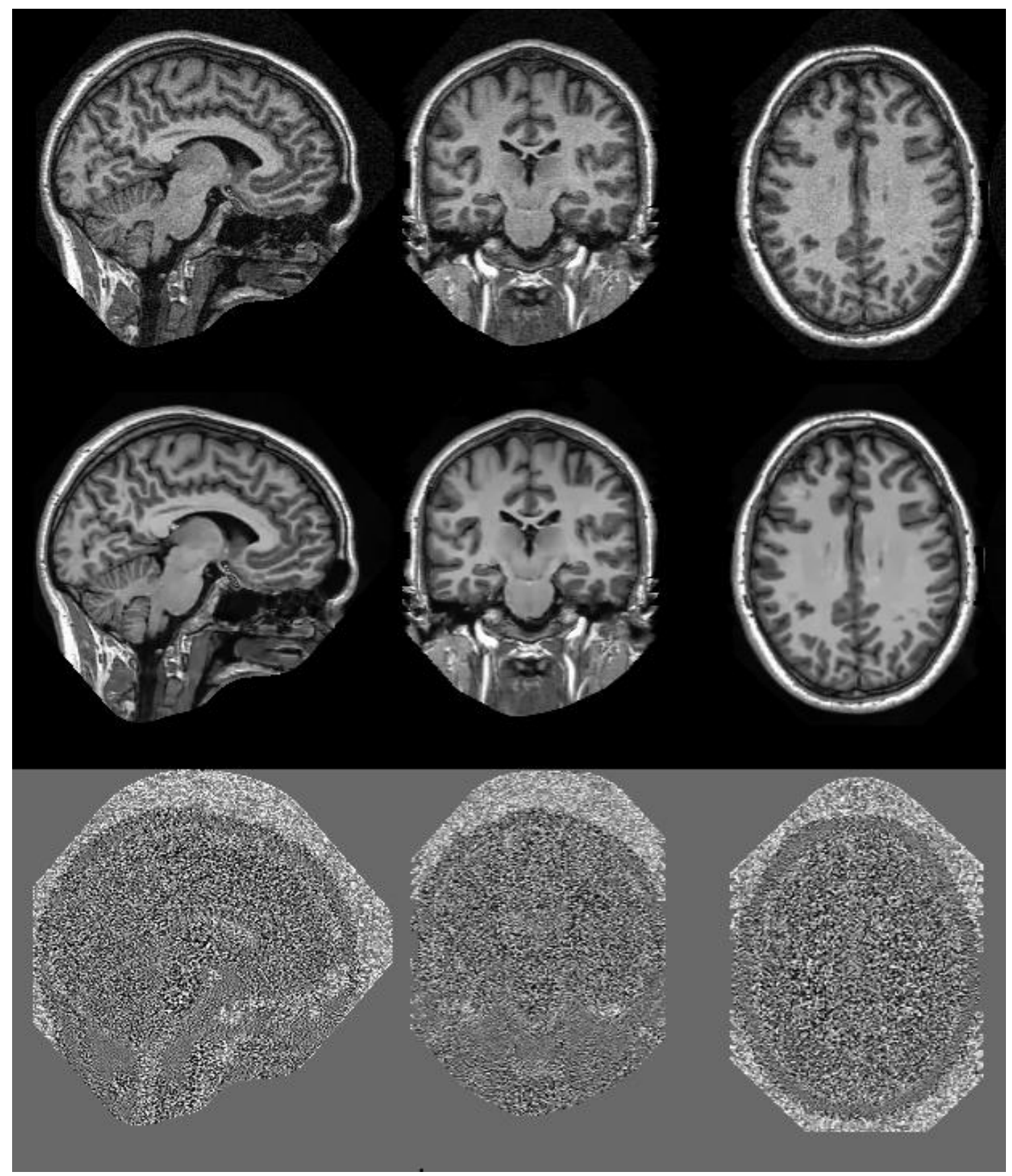

Fig. 8. Example results of the proposed PRI-NLM3D filter on real data (Rician noise level of $2 \%$ ). The background and part of the face were removed by a defacer program to preserve the anonymity of the subject. From top to bottom: Original noise volume, denoised volume using the proposed method, and the corresponding residuals. 
PRI-NLM3D

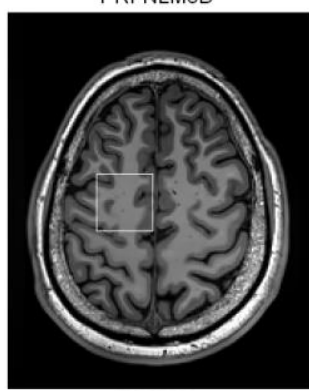

ODCT3D

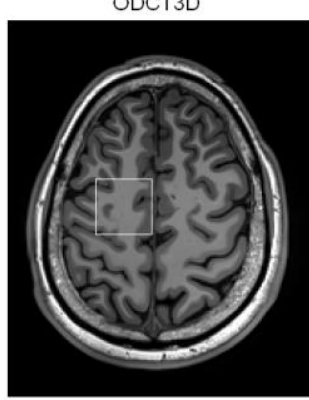

Original

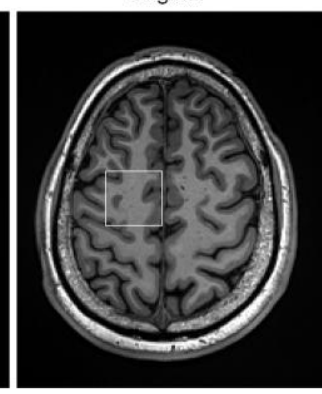

ORNRAD

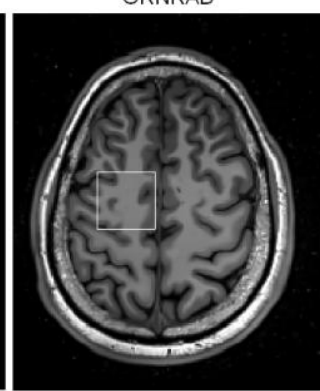

PRI-NLM3D

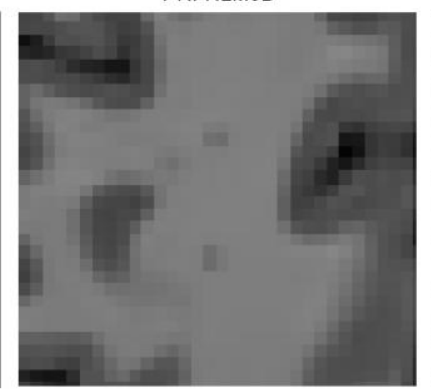

ODCT3D

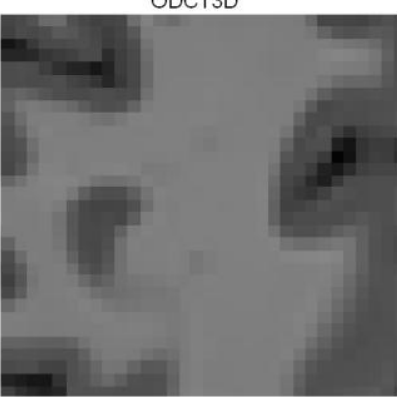

Original

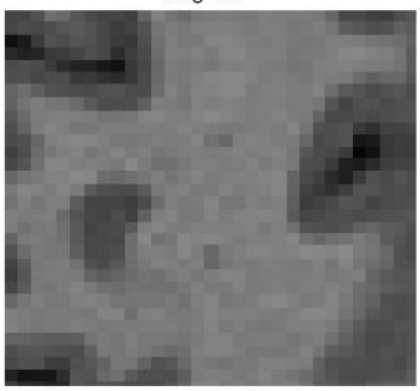

ORNRAD

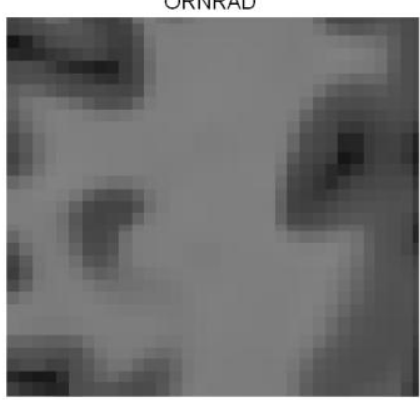

Fig. 9. Example results of filters applied to real data (Rician noise level of 1\%). All compared methods worked very well, but the ODCT3D, and especially the ORNRAD method, tended to oversmooth some fine details in the images (see magnification of the white square region in the lower row).

\section{Discussion}

We presented two new methods for MRI denoising (ODCT3D and PRI-NLM3D) that take advantage of two intrinsic properties of MR images: sparseness and self-similarity. The proposed PRI-NLM3D method was compared with state-ofthe-art methods in MRI denoising and produced the best results out of all the methods under consideration.

The enhanced accuracy of the proposed methods can be understood by taking two main facts into consideration. First, the ODCT3D method benefits from the high compressibility (sparseness) of MRI data, allowing a very efficient noise reduction (the ODCT3D method obtained very good results in comparison with previously proposed NLM-based methods). Second, the PRI-NLM3D method indirectly takes benefit from the sparseness properties of the images by using the prefiltered ODCT3D data as prior. This method showed a very good performance as a result of using the low noise image prior and the increased number of redundant patterns present when using a rotationally invariant 
similarity measure (compared with the non-rotationally invariant NLM voxelwise version). It is worth noting that, although other nonlocal rotationally invariant methods have been previously proposed in the literature, they were $2 \mathrm{D}$ implementations rather than 3D like the one proposed in this paper.

Both the ODCT3D and PRI-NLM3D methods showed an improved performance over previous state-of-the-art MRI denoising methods. The main differences between these two methods exist in the small details: The ODCT3D method tends to slightly oversmooth edges and some fine details (compared with the PRI-NLM3D), probably as a result of its nature as a block processor, whereas the PRI-NLM3D method seems to retain more detail in the denoised image because of its voxelwise processing.

From a practical point of view, the proposed methods run in a reasonable time (less than $1 \mathrm{~min}$ ) for most research and clinical settings. Moreover, GPU-based implementations can be used to further reduce the processing time. Recently, a GPU-based NLM filter implementation showed a drastic reduction in the computational burden (Huang et al., 2009), which could allow for an almost real-time version of the proposed filters.

Finally, it is worth noting that the application of the proposed method can clearly benefit not only visual diagnostics, but also quantitative methodologies that rely on good quality data, such as MRI brain tissue segmentation or MR diffusion tensor image processing.

\section{Acknowledgments}

We are grateful to Dr. Krissian for providing us with the results of his denoising method for the comparison section. This work has been partially supported by the Spanish Health Institute Carlos III through the RETICS Combiomed, RD07/0067/2001, the Spanish Ministry Science and Innovation through grant TIN2008-04752, and the Canadian Institutes of Health Research Industry Cda (CECR)-Gevas-OE016. 


\section{References}

Aharon M, Elad M and Bruckstein A.M. 2006. K-SVD: an algorithm for designing overcomplete dictionaries for sparse representation. IEEE Trans. Signal Process, 54, 4311-4322.

Ashburner J. and Friston K.J. 2000. Voxel-based morphometry-the methods, Neurolmage, 11, 805-821.

Buades A, Coll B and Morel J.M. 2005. A non-local algorithm for image denoising. IEEE Int. Conf. on Computer Vision and Pattern Recognition (CPVR) 2, 60-65.

Collins D.L., Zijdenbos A.P., Kollokian V., Sled J.G., Kabani N.J., Holmes C.J. and Evans A.C. 1998. Design and construction of a realistic digital brain phantom. IEEE Trans. Med. Imaging, 17, 463-468.

Coupé P., Yger P., Prima S., Hellier P., Kervrann C. and Barillot C. 2008a. An optimized blockwise nonlocal means denoising filter for 3-D magnetic resonance images. IEEE Trans. Med. Imaging, 27, 425-441.

Coupé P., Hellier P., Prima S., Kervrann C. and Barillot C. 2008b. 3D wavelet sub-bands mixing for image denoising. Int. J. Biomed. Imaging, Article ID: 590183. doi:10.1155/2008/590183.

Coupé P., Manjon J.V., Gedamu E., Arnold D., Robles M. and Collins D.L. 2010. Robust Rician noise estimation for MR images. Med. Image Anal, 14, 483-493.

Elad M. and Aharon M. 2006. Image denoising via learned dictionaries and sparse representation. Proc. IEEE Computer Soc. Conf. on CPVR 1, 895-900. 
Gerig G., Kubler O., Kikinis R. and Jolesz F.A. 1992. Nonlinear anisotropic filtering of MRI data. IEEE Trans. Med. Imaging, 11, 221-232.

Guleryuz O.G. 2003. Weighted overcomplete denoising. Proc. Asilomar Conf. on Signals and Systems.

Guleryuz O.G. 2007. Weighted averaging for denoising with overcomplete dictionaries. IEEE Trans. Image Process, 16, 3020-3034.

Hu M.K. 1962. Visual pattern recognition by moment invariants. IRE Trans. Info. Theory, 8, 179-187.

Huang K., Zhang D. and Wang K. 2009. Non-local means denoising algorithm accelerated by GPU. Proc. Soc. of Photo-Optical Instrumentation Engineers (SPIE), 7497.

Kervrann C., Boulanger J. and Coupé P. 2007. Bayesian non-local means filter, image redundancy and adaptive dictionaries for noise removal. Proc. Conf. Scale Space and Variational Meth. in Computer Vision, 520-532.

Kleinschmidt O., Brox T. and Cremers D. 2008. Nonlocal texture filtering with efficient tree structures and invariant patch similarity measures. Proc. Int. Workshop on Local and Non-Local Approximation in Image Process, LNLA2008.

Krissian K. and Aja-Fernandez S. 2009. Noise-driven anisotropic diffusion filtering of MRI. IEEE Trans. Image Process, 18, 2265-2274.

Kwan R.K.-S., Evans A.C. and Pike G.B. 1999. MRI simulation-based evaluation of image-processing and classification methods. IEEE Trans. Med. Imaging, 18, 1085-1097. 
Lou Y., Favaro P. and Soatto S. 2008. Nonlocal similarity image filtering. Research Reports CAM (8-26).

Mallat S. 1999. A wavelet tour of signal processing, Academic Press.

Manjón J.V., Carbonell-Caballero J., Lull J.J., Garcia-Martí G., Martí-Bonmatí L. and Robles M. 2008. MRI denoising using non-local means. Med. Image Anal, 4 , 514-523.

Manjón J.V., Coupé P., Martí-Bonmatí L., Robles M. and Collins D.L. 2010. Adaptive non-local means denoising of MR images with spatially varying noise levels. J. Magn. Reson. Imaging, 31, 192-203.

Mairal J., Sapiro G. and Elad M. 2008. Learning multiscale sparse representations for image and video restoration. Multiscale Model. Simul., 7, 214-241.

Muresan D.D. and Parks T.W. 2003. Adaptive principal components and image denoising. IEEE Int. Conf. Image Process., 1, 101-104.

Nowak R. 1999. Wavelet-based Rician noise removal for magnetic resonance imaging. IEEE Trans. Image Process, 8, 1408-1419.

Pizurica A., Philips W., Lemahieu I. and Acheroy M. 2003. A versatile wavelet domain noise filtration technique for medical imaging. IEEE Trans. Med. Imaging, 22, 323-331.

Teague M.R. 1980. Image analysis via the general theory of moments. J. Opt. Soc. Am. $70,920-930$.

Wang Z., Bovik A.C., Sheikh H.R. and Simoncelli E.P. 2004. Image quality assessment: from error visibility to structural similarity. IEEE Trans. Image Process., 13, 600-612. 
Wiest-Daesslé N., Prima S., Coupé P., Morrissey S.P. and Barillot C. 2008. Rician noise removal by non-local means filtering for low signal-to-noise ratio MRI: applications to DR-MRI. MICCAI, 11, 171-179.

Yaroslavsky L.P., Egiazarian K. and Astola J. 2000. Transform domain image restoration methods: review, comparison and interpretation. TICSP Series \#9, TUT, Tampere, Finland, ISBN 952-15-0471-4.

Zexuan J., Qiang C., Quan-Sen S. and De-Shen X. 2009. A moment-based nonlocal-means algorithm for image denoising. Information Processing Letters $109,1238-1244$.

Zimmer S., Didas S. and Weickert J.S. 2008. A rotationally invariant block matching strategy improving image denoising with non-local means. Proc. LNLA-2008. 